\title{
Multistep Ionization of Argon Clusters in Intense Femtosecond Extreme Ultraviolet Pulses
}

\author{
C. Bostedt, ${ }^{*}$ H. Thomas, M. Hoener, and E. Eremina \\ Institut für Optik und Atomare Physik, Technische Universität Berlin, \\ Eugene-Wigner-Bldg. EW 3-1, Hardenbergstr. 36, 10623 Berlin, Germany
}

T. Fennel and K.-H. Meiwes-Broer

Institut für Physik, Universität Rostock, 18051 Rostock, Germany

H. Wabnitz, M. Kuhlmann, E. Plönjes, K. Tiedtke, R. Treusch, and J. Feldhaus

Deutsches Elektronen-Synchrotron DESY, 22603 Hamburg, Germany

\author{
A. R. B. de Castro \\ Laboratorio Nacional de Luz Sincrotron, 13084-971 Campinas SP, Brazil \\ and Instituto de Fisica Gleb Wataghin, Universidade Estadual de Campinas, 13083-970 Campinas SP, Brazil
}

T. Möller

Institut für Optik und Atomare Physik, Technische Universität Berlin, Eugene-Wigner-Bldg. EW 3-2, Hardenbergstr. 36, 10623 Berlin, Germany

(Received 8 October 2007; published 1 April 2008)

\begin{abstract}
The interaction of intense extreme ultraviolet femtosecond laser pulses $(\lambda=32.8 \mathrm{~nm})$ from the FLASH free electron laser (FEL) with clusters has been investigated by means of photoelectron spectroscopy and modeled by Monte Carlo simulations. For laser intensities up to $5 \times 10^{13} \mathrm{~W} / \mathrm{cm}^{2}$, we find that the cluster ionization process is a sequence of direct electron emission events in a developing Coulomb field. A nanoplasma is formed only at the highest investigated power densities where ionization is frustrated due to the deep cluster potential. In contrast with earlier studies in the IR and vacuum ultraviolet spectral regime, we find no evidence for electron emission from plasma heating processes.
\end{abstract}

DOI: 10.1103/PhysRevLett.100.133401

The interaction of intense laser pulses with matter is an issue of fundamental interest and only scarcely investigated in the spectral regime of $\lambda \leq 100 \mathrm{~nm}$. One of the fundamental questions in this context is how ionization and absorption properties of complex systems develop in the vacuum ultraviolet (VUV) and extreme ultraviolet (XUV) strong-field domain. For such investigations, atomic clusters are ideal targets as hidden energy dissipation into surrounding media is virtually absent.

Previously, much effort has been put into the study of clusters in intense infrared (IR) laser pulses where the efficient coupling to a transient nanoplasma results in extremely high absorption. For rare-gas clusters, which are used in most experiments, the nanoplasma buildup (inner ionization [1]) is initiated by optical field ionization and subsequently supported by electron impact ionization. The energy capture leading to the removal of cluster electrons (outer ionization [1]) is of plasma nature and mostly driven by inverse bremsstrahlung (IBS). The absorption as well as electron emission can be further enhanced through resonant plasmon excitations [2,3]. At short wavelengths $\lambda \leq 100 \mathrm{~nm}$, however, collective effects can be disregarded as the required critical density cannot be reached [4]. Further, due to the $\omega^{-2}$ scaling of the cross section for IBS, strongly reduced absorption efficiency was expected
PACS numbers: $36.40 . \mathrm{Gk}, 02.50 . \mathrm{Ng}, 41.60 . \mathrm{Cr}, 52.50 . \mathrm{Jm}$

in this regime. Because of that, it was a big surprise that complete Coulomb explosion, highly charged ions [5], and thermionic electron emission [6] were observed in first VUV experiments at $\lambda=100 \mathrm{~nm}$ on $\mathrm{Xe}_{N}$ at intensities of $10^{13} \mathrm{~W} / \mathrm{cm}^{2}$. These results sparked significant theoretical efforts [7-10]. For the description of the absorption and cluster ionization, new concepts were introduced, such as more realistic potentials for IBS [7,8], barrier suppression in the ionized cluster [9], and enhanced heating through many-body collisions in a transient strongly coupled nanoplasma [10]. Recent calculations predicted that IBS even remains the dominant energy absorption process for wavelength down to $62 \mathrm{~nm}$ [11].

In this Letter, we present the first data from intense XUV laser pulse-cluster interactions far above the ionization threshold at $\lambda=32.8 \mathrm{~nm}(h \nu=37.8 \mathrm{eV})$ and intensities up to $5 \times 10^{13} \mathrm{~W} / \mathrm{cm}^{2}$. The experiments are performed on $\operatorname{Ar}_{N}(N \sim 100)$ and analyzed with photoelectron spectroscopy (PES). The electron energy distribution provides insight into the coupling between the photon field and the cluster $[6,12-14]$. By comparing the experimental results to complementary Monte Carlo simulations, three key findings have been worked out:

First, the cluster ionization is a multistep process of direct electron emission events driven by single photon 
absorption. Second, the energy downshift for subsequently released electrons due to the space charge buildup results in an a highly nonthermal electron energy distribution. Third, a nanoplasma develops only for the highest investigated intensities where the cluster potential frustrates further direct electron emission. Also in this case, no discernible signatures from plasma heating are observed.

Argon has been chosen as target material because of its flat atomic photoionization cross section in the XUV spectral regime. The sample jets are prepared by expanding argon through a $100 \mu \mathrm{m}$ conical nozzle in front of a pulsed valve. The resulting cluster or atomic jet crosses the free electron laser (FEL) beam below the aperture of a field-free time-of-flight (tof) electron spectrometer. The FEL is operated in single-bunch mode, delivering pulse energies up to $25 \mu \mathrm{J}$ with pulse durations of $25 \mathrm{fs}$ [15]. The experiments are performed at the FLASH micro focus beam line BL2 with a focal spot diameter of $50 \mu \mathrm{m}$ FWHM, resulting in peak intensities up to $5 \times 10^{13} \mathrm{~W} / \mathrm{cm}^{2}$, similar to previous VUV experiments $[5,6]$. To increase the signal contrast between cluster and atomic contributions from residual gas, the tof detector is mounted perpendicular to the polarization plane of the incoming light. Compared to atoms the photoemission from clusters is much more isotropic [16].

Figure 1 displays the electron data for Ar clusters and atoms at pulse intensities of $3 \times 10^{13} \mathrm{~W} / \mathrm{cm}^{2}$. The atomic single-shot spectra (inset of Fig. 1) show only one peak at $57 \mathrm{~ns}$ from the Ar $3 p$ photoemission. For the clusters, additional peaks towards longer flight times appear, indi-

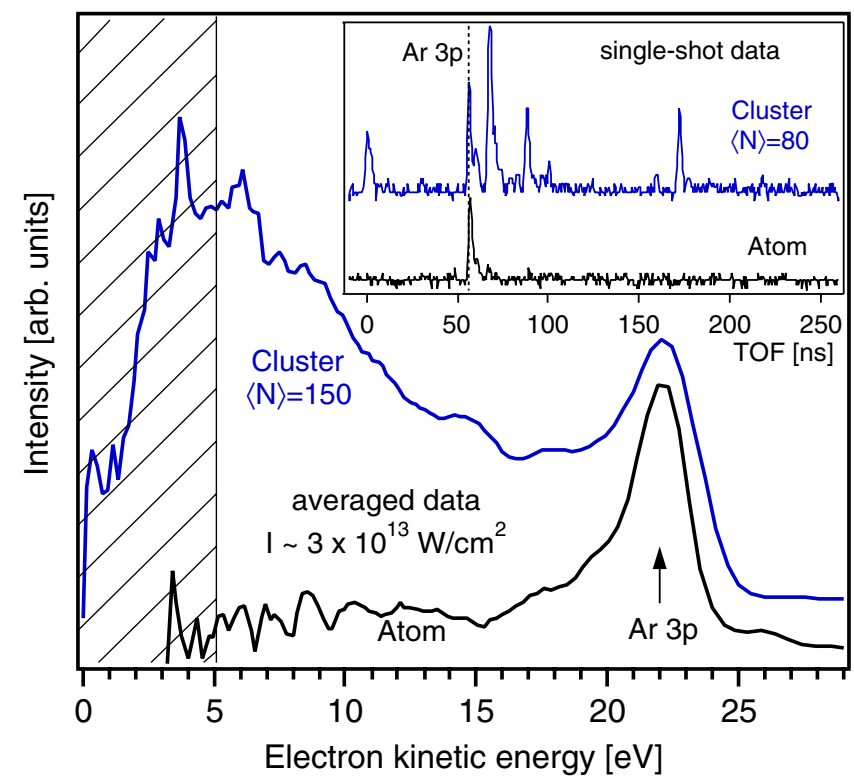

FIG. 1 (color online). Photoelectron spectra converted from the single-shot tof data (inset) for Ar atoms (bottom) and clusters (top) as explained in the text. In the averaged photoelectron spectra, the cluster shows continuous electron intensity towards lower kinetic energies. The signal below $5 \mathrm{eV}$ is affected by the strongly decreasing detector transmission function. cating a series of subsequent ionization events. Electrons at flight times below $57 \mathrm{~ns}$ are virtually absent. The decreasing electron kinetic energies within this multistep ionization process reflect the developing Coulomb cluster potential [17]. The observation of a few single photoelectron events in the cluster spectrum is in good agreement with statistical photoemission into the covered solid angle element of the detector under the current experimental conditions $\sigma_{3 p}=5$ Mbarn, $\beta_{3 p}=0.8, I=3 \times 10^{13} \mathrm{~W} / \mathrm{cm}^{2}$ ) and a detection efficiency of $\leq 10 \%$.

To obtain the photoelectron spectra in Fig. 1, the singleshot data are sorted into bins according to the FEL pulse energies, averaged, and converted to a kinetic energy scale. Typically, 1000 scans are contained in each bin. For clarity, the spectra are smoothed over $0.25 \mathrm{eV}$ energy windows below $15 \mathrm{eV}$. In the atom spectra in Fig. 1, the Ar $3 p$ photoemission at $22 \mathrm{eV}$ is the only significant feature and exhibits a width of $2.3 \mathrm{eV}$ (FWHM) due to the $3 \mathrm{~ns}$ time resolution of the detector. The cluster spectrum shows a very different electron distribution. Starting at the right hand side of the spectra, it also contains the prominent Ar $3 p$ photoemission line but never drops back to the baseline as in the atomic case. Instead, a continuous electron signal towards lower energies is observed. Except for the Ar $3 p$ main line, all other peaks observable in the single-shot data completely average out, suggesting a statistical photoemission process from the cluster.

Figure 2 displays a corresponding ion tof spectrum. In contrast to earlier investigations in the VUV [5] and IR [18] spectral regime, the clusters do not disintegrate completely. The most dominant signal is the Ar monomer.

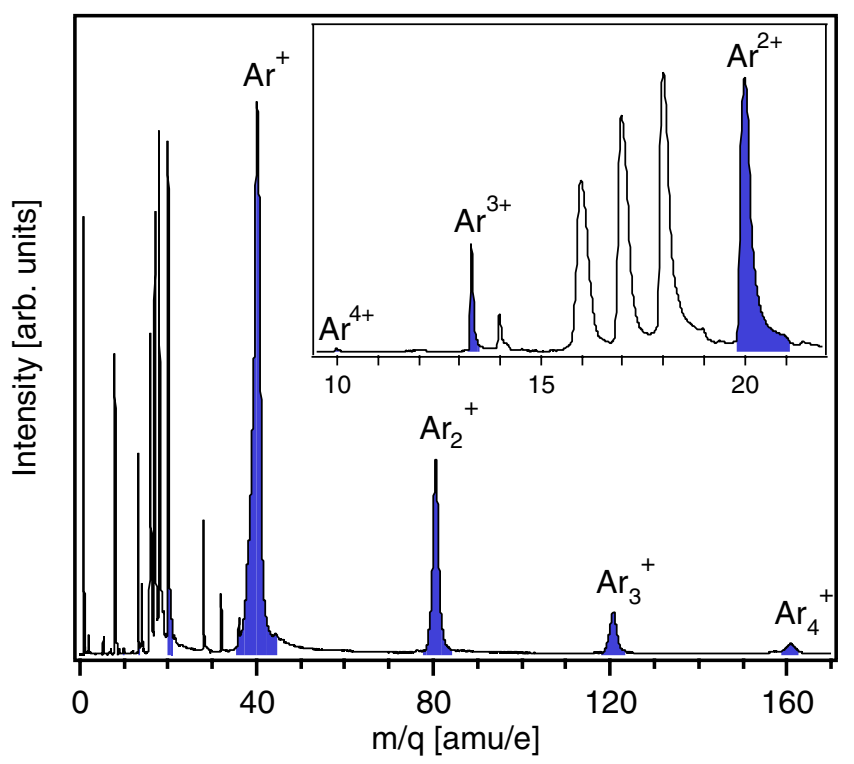

FIG. 2 (color online). Time-of-flight mass spectra of Ar clusters (shaded signal) with the lower $m / q$ ratios shown in more detail in the inset for clarity. The dominant signal stems from monomers and larger cluster fragments. All Ar fragments exhibit rather narrow peaks indicating that they do not carry significant kinetic energies. 
Larger fragments as well as moderately high charge states up to $\mathrm{Ar}^{4+}$ can be observed. The $\mathrm{Ar}^{+}$fragments exhibit low average kinetic energies of only up to $30 \mathrm{eV}$ indicating a much less severe Coulomb explosion and less efficient electron removal from the cluster compared to experiments at longer wavelength $[5,18]$.

For comparison, photoelectron spectra are simulated assuming statistical ionization of the constituents with the atomic photoionization cross sections at $\lambda=32.8 \mathrm{~nm}$. The cross sections are taken to be 5 and 0.1 Mbarn for the Ar $3 p$ and $3 s$ photoionization and 2.6 Mbarn for $\mathrm{Ar}^{+}$, respectively [19]. Because the resulting average atomic charge states and kinetic energies (Fig. 1) are much lower compared to earlier modeling on Coulomb explosion of molecules [20], ionic motion during the pulse can be neglected. The escape of electrons is described as an instantaneous process; i.e., the freed electrons leave the cluster before the next ionization event occurs. The asymptotic kinetic energy of an electron released from the $j$-th ion is determined from

$$
E_{k}=h \nu-I_{p}-\frac{e^{2}}{4 \pi \epsilon_{0}} \sum_{i \neq j} \frac{q_{i}}{r_{i j}},
$$

where $I_{p}$ is the ionization potential and $i$ runs over all other ions with charge state $q_{i}$ and distance $r_{i j}$. To calculate the photoelectron spectra, only direct ionization events, i.e., electrons with $E_{k}>0$, are accepted. The resulting energy spectra are typically averaged over $10^{4}$ simulations to represent a statistical energy spectrum.

Figure 3 shows each ionization step as well as the summed spectrum of $\mathrm{Ar}_{147}$ for a laser intensity of $I=3 \times$ $10^{13} \mathrm{~W} / \mathrm{cm}^{2}$, similar to the experimental conditions in Fig. 1. The first spectrum represents the photoemission of a cluster in the single photon regime [16]. The prominent Ar $3 p$ line at $22 \mathrm{eV}$ plus the 50 times weaker Ar $3 s$ contribution can be identified. Already for the second photoelectron, the spectrum changes significantly. Focusing the

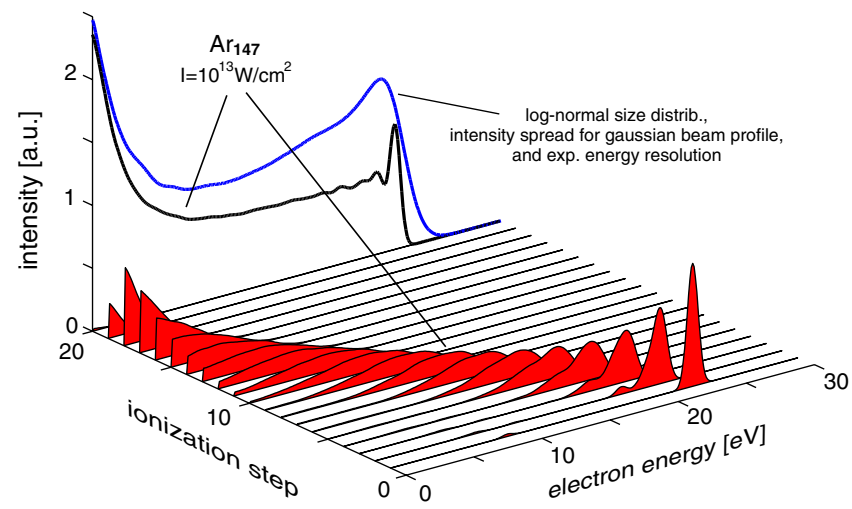

FIG. 3 (color online). Monte Carlo simulations of the electron emission from an $\mathrm{Ar}_{147}$ cluster as described in the text. The averaged spectra of each emitted electron numbers (filled graphs) as well as their average (black line) are shown. For comparison with the data, the relevant broadening effects are considered. discussion on the dominant Ar $3 p$ level, the peak becomes broader and develops substructure. This effect is due the Coulomb field of the ion produced previously. For an increasing ionization, the spectrum becomes broader and shifts to lower kinetic energies due to further field buildup. After about 10 ionization steps, only electrons produced in regions with sufficiently weak potential can escape from the cluster, and the photoelectron signal starts being cut at zero kinetic energies (partial frustration of direct photoemission). After about 19 ionization steps, the strong Coulomb field leads to total frustration of direct ionization for this cluster size. The sum over all single emission spectra is shown as a solid black line. Further, the intensity spread of a Gaussian beam profile, the experimental resolution, and the cluster size distribution are considered for comparison with the experimental data. The spectrum including all effects is shown on top of the average line in Fig. 3, showing a remarkable similarity to the experimental data in Fig. 1. In particular, the prominent $\mathrm{Ar} 3 p$ peak as well as the continuous spectral intensity at lower kinetic energies are reproduced.

More detailed information can be extracted from the intensity dependent spectra. Figure 4 displays the experimental results from $\operatorname{Ar}_{N}$ with $\langle N\rangle=80$. The laser power is varied between $2 \times 10^{11}-5 \times 10^{13} \mathrm{~W} / \mathrm{cm}^{2}$ by driving the

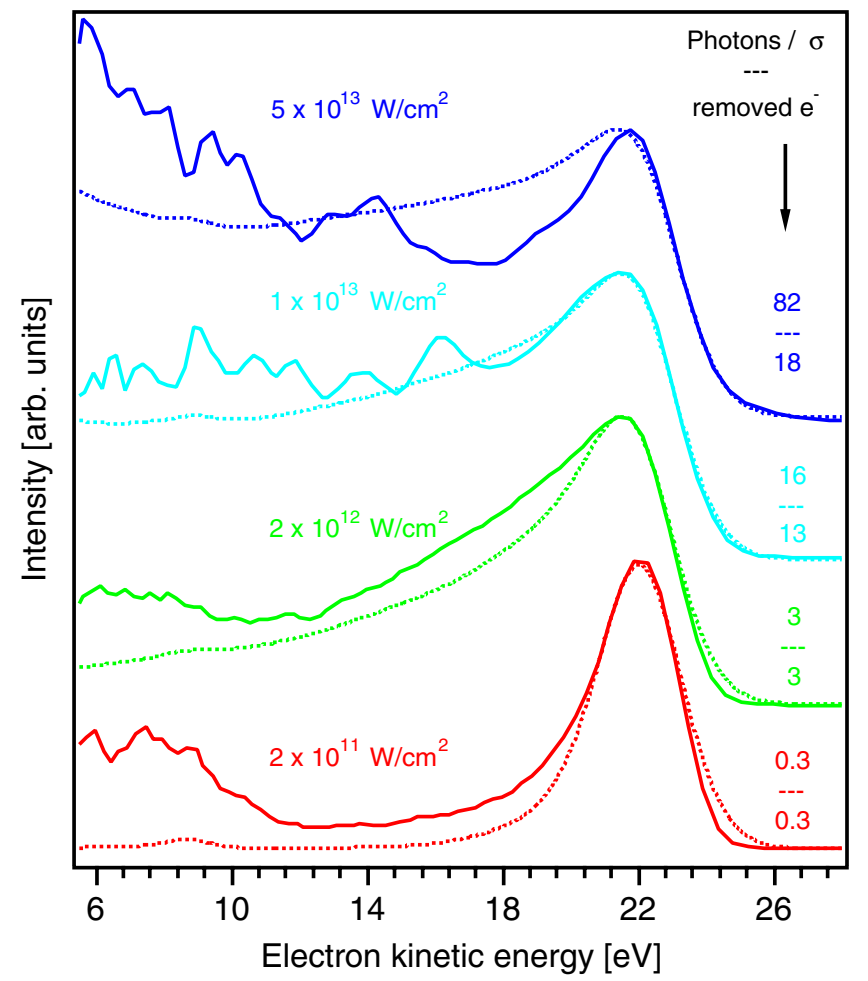

FIG. 4 (color online). Experimental (solid line) and modeled (dashed line) electron spectra for $\mathrm{Ar}_{80}$ clusters for increasing power densities from bottom to top. The numbers on the right hand side indicate how many photons fall into the absorption cross section of the cluster and how many electrons can overcome the increasing Coulomb barrier. 
experimental setup out of the laser focus and sorting the data according to the incoming pulse energies. Additionally, the modeled energy spectra calculated as described above and the average number of removed electrons are added to Fig. 4. For the lowest power densities of $2 \times$ $10^{11} \mathrm{~W} / \mathrm{cm}^{2}$ on average, only 0.3 photons fall into the absorption cross section of the cluster. The corresponding spectra resemble the single photon limit [16]. At the next higher power density of $2 \times 10^{12} \mathrm{~W} / \mathrm{cm}^{2}$, the transition region from single to multistep ionization is probed. Here, three photons fall into the absorption cross section of the cluster leading to asymmetric broadening of the $3 p$ photoemission line to lower energies. All photoelectrons can still leave the cluster but are increasingly slowed by the cluster potential. This trend continues up to $1 \times 10^{13} \mathrm{~W} / \mathrm{cm}^{2}$ where the next critical point is reached. Here, the number of absorbed photons becomes comparatively larger than the number of electrons that can leave the cluster and frustration of the photoemission begins. As a result, the lowenergy part of the spectrum turns into a plateau. For the highest investigated power densities of $5 \times 10^{13} \mathrm{~W} / \mathrm{cm}^{2}$, over 80 photons are absorbed by the cluster. However, after the emission of 18 electrons, the large space charge frustrates all further direct photoemission. Instead, additional photon absorption creates quasifree electrons that are trapped in the cluster potential, and a nanoplasma develops. For these highest investigated power densities, the data shows higher spectral intensity towards low kinetic energies than the multistep model. Possible explanations for this discrepancy may be thermalization of nanoplasma electrons or plasma heating. Plasma heating has been predicted to still be the dominant absorption mechanism for $62 \mathrm{~nm}$ pulses with similar power densities, resulting in a Maxwell-Boltzmann electron distribution [11]. In the current data, the formation of a thermal high-energy tail above the $\operatorname{Ar} 3 p$ level cannot be observed. Therefore, it must be concluded that even for the highest power densities, emission from a laser-heated nanoplasma is not significant, and the multistep ionization process is dominant. It should be mentioned that for the calculations in Ref. [11], a much longer pulse (100 fs vs $25 \mathrm{fs})$ is assumed and that the plasma heating starts only after the ionization has saturated about 40 fs into the pulse, further underlining that in the present experiments, IBS has no significant effect.

In conclusion, we have studied the photoemission of $\mathrm{Ar}$ clusters in intense XUV $(h \nu=37.8 \mathrm{eV})$ laser pulses experimentally by photoelectron spectroscopy and theoretically by Monte Carlo simulations. Our analysis reveals that the electron emission is driven by direct multistep ionization, in contrast to the cooperative scheme of inner ionization and subsequent nanoplasma heating observed in the IR and VUV spectral regime. Within the multistep ionization process, the residual cluster Coulomb field reduces the kinetic energy of subsequently released electrons and eventually frustrates further direct emission, leading to the generation of a nanoplasma. However, heating of the nanoplasma appears to be of minor importance. The frustration effect within the multistep ionization will be of great importance for all near-threshold excitations of clusters and large molecules in the short-wavelength strong-field domain.

We would like to thank all DESY staff for their outstanding support. Funding is acknowledged from BMBF No. 05KS4KTC1, DFG Sonderforschungsbereich No. 652, and HGF Virtuelles Institut VH-VI-103.

*Corresponding author: bostedt@physik.tu-berlin.de

[1] I. Last and J. Jortner, Phys. Rev. A 62, 013201 (2000).

[2] J. Zweiback, T. Ditmire, and M. D. Perry, Phys. Rev. A 59, R3166 (1999).

[3] T. Fennel, T. Döppner, J. Passig, C. Schaal, J. Tiggesbäumker, and K.-H. Meiwes-Broer, Phys. Rev. Lett. 98, 143401 (2007).

[4] H. M. Milchberg, S. J. McNaught, and E. Parra, Phys. Rev. E 64, 056402 (2001).

[5] H. Wabnitz, L. Bittner, A. R. B. de Castro, R. Döhrmann, P. Gürtler, T. Laarmann, W. Laasch, J. Schulz, A. Swiderski, and K. von Haeften et al., Nature (London) 420, 482 (2002).

[6] T. Laarmann, M. Rusek, H. Wabnitz, J. Schulz, A. R. B. de Castro, P. Gürtler, W. Laasch, and T. Möller, Phys. Rev. Lett. 95, 063402 (2005).

[7] R. Santra and C. H. Greene, Phys. Rev. Lett. 91, 233401 (2003).

[8] Z. B. Walters, R. Santra, and C. H. Greene, Phys. Rev. A 74, 043204 (2006).

[9] C. Siedschlag and J. M. Rost, Phys. Rev. Lett. 93, 043402 (2004).

[10] C. Jungreuthmayer, L. Ramunno, J. Zhanghellini, and T. Brabec, J. Phys. B 38, 3029 (2005).

[11] I. Georgescu, U. Saalmann, and J. M. Rost, Phys. Rev. A 76, 043203 (2007).

[12] Y. L. Shao, T. Ditmire, J. W. G. Tisch, E. Springate, J.P. Marangos, and M.H. R. Hutchinson, Phys. Rev. Lett. 77, 3343 (1996).

[13] V. Kumarappan, M. Krishnamurthy, and D. Mathur, Phys. Rev. A 66, 033203 (2002).

[14] E. Springate, S. A. Aseyev, S. Zamith, and M. J.J. Vrakking, Phys. Rev. A 68, 053201 (2003).

[15] W. Ackermann et al., 1, 336 (2007).

[16] D. Rolles, H. Zhang, Z. D. Pesic, R. C. Bilodeau, A. Wills, E. Kukk, B.S. Rude, G. D. Ackermann, J. D. Bozek, and R. Diez Muino et al., Phys. Rev. A 75, 031201(R) (2007).

[17] U. Näher, H. Göhlich, T. Lange, and T. P. Martin, Phys. Rev. Lett. 68, 3416 (1992).

[18] T. Ditmire, R. A. Smith, J.W. G. Tisch, and M.H. R. Hutchinson, Phys. Rev. Lett. 78, 3121 (1997).

[19] A. M. Covington, A. Aguilar, I. Alvarez, C. Cisneros, I. R. Covington, I. Dominguez, M. Gharaibeh, G. Hinojosa, M.M. SantAnna, and R.A. Phaneuf et al., XXII International Conference on Photonic, Electronic and Atomic Collisions, Santa Fe, New Mexico, Abstracts of Contributed Papers (2001), p. 48.

[20] R. Neutze, R. Wouts, D. van der Spoel, E. Weckert, and J. Hajdu, Nature (London) 406, 752 (2000). 